\title{
Parameter Identification of Fractional Order Nonlinear System Based on Haar Wavelet Operational Matrix
}

\author{
Yuanlu $\mathrm{Li}^{1,2}$, Min Jiang ${ }^{1, *}$ and ${\mathrm{Jun} \mathrm{Li}^{1}}^{1}$ \\ ${ }^{1}$ B-DAT, School of Information and Control, Nanjing University of Information Science \& Technology, Nanjing, China, 210044 \\ ${ }^{2}$ Jiangsu Collaborative Innovation Center on Atmospheric Environment and Equipment Technology, Nanjing University of \\ Information Science \& Technology, Nanjing, China, 210044 \\ ${ }^{*}$ Corresponding author
}

\begin{abstract}
A parameter identification method for fractional order nonlinear systems was proposed. The basic idea is to use the Haar wavelets to represent the input and output signals, and then convert the nonlinear differential into a corresponding integral equation. As a result, the parameters of the nonlinear system are determined by minimizing the errors between the output of the real system and that of the identified system. An advantage of the proposed method lies in fastening the identification process by using the multi-resolution nature of the wavelet.
\end{abstract}

Keywords-nonlinear system; system identification; Haar wavelet; operational matrix

\section{INTRODUCTION}

A fractional order system (FOS) is a system governed by a fractional differential equation. A growing number of physical systems can be concisely described by FOS, such as the semiinfinite lossy (RC) transmission line [1, 2], diffusion of heat into semi-infinite solid [2], viscoelastic materials [3]. Duffing oscillator as a classical dynamical model has been intensively studied with fractional order derivative [4].

Hence, we mainly consider the fractional order nonlinear system described by:

$$
{ }_{0} D_{t}^{2} y(t)+c_{0} D_{t}^{\alpha} y(t)+k y(t)+\lambda y^{3}(t)=f(t) .
$$

with the initial condition

$$
y(0)=d_{0}, y^{\prime}(0)=d_{1}
$$

where $c, k$ and $\lambda$ are real constant coefficients, also $0<\alpha<2$, and ${ }_{0} D_{t}^{\alpha}$ denotes the Caputo fractional derivative of order $\alpha$. $f(t)$ is the excitation signal and $y(t)$ is the output response of the system. Obviously, when $\alpha=1$, (1) turns back into the classical Duffing oscillator.

Because the geometric and physical interpretation of fractional calculus is not as clear as integer calculus, it is difficult to directly model real systems with FOS based on mechanism analysis. Therefore, system identification is a practical way to model a real system with FOS. However, because of the existence of fractional order derivatives in the system, the identification process of a FOS is more complex than that of an integral order system [5]. Therefore, most classical identification methods cannot directly applied to the identification of a FOS.

In this paper, the Haar wavelet operational matrix was proposed to identify the nonlinear FOS. The mainly reason for this is that operational matrix has been widely used to deal with FOS. The main feature of this approach is that it converts the FOS into a system of algebraic equation. Thus the problem was simplified. Typical examples include the block-pulse functions [6], Chebyshev polynomials [7] and Haar wavelets [8].

The organization of this paper is as follows: in Section II, the fundamental definition of fractional calculus is presented. In Section III, we outline the identification method based on Haar wavelet operational matrix. The verification of the identification method is provided in Section IV. Finally, conclusions are drawn in Section V.

\section{PRELIMINARIES AND NOTATIONS}

\section{A. Fractional Calculus}

There are several definitions for fractional calculus. Here the Riemann-Liouville fractional integral and Caputo fractional derivative are given as following, which will be used in this paper.

The Riemann-Liouville fractional integration of order $\alpha>0$ is defined as

$$
\left(I^{\alpha} f\right)(t)=\frac{1}{\Gamma(\alpha)} \int_{0}^{t}(t-\tau)^{\alpha-1} f(\tau) d \tau=\frac{1}{\Gamma(\alpha)} t^{\alpha-1} * f(t)
$$

where $\Gamma$ is the Gamma function.

The Caputo’s derivative is defined by 


$$
\left(D^{\alpha} f\right)(t)=\frac{1}{\Gamma(n-\alpha)} \int_{0}^{t} \frac{f^{(n)}(\tau)}{(t-\tau)^{\alpha+1-n}} d \tau
$$

where $n-1<\alpha \leq n$ and $n$ is an integer.

The relationship between the Riemann-Liouville integral and Caputo derivative is given by

$$
\left(I^{\alpha} D^{\alpha} f\right)(t)=f(t)-\sum_{k=0}^{n-1} f^{(k)}\left(0^{+}\right) \frac{t^{k}}{k !} .
$$

where $n-1<\alpha \leq n$ and $n$ is an integer.

\section{B. Haar Wavelet}

The Haar wavelets are defined as following:

$$
\begin{aligned}
& h_{n}=h_{1}\left(2^{j} t-k\right), \quad n=2^{j}+k, \\
& j \geq 0, \quad 0 \leq k \leq 2^{j}, \quad n, j, k \in Z .
\end{aligned}
$$

where

$$
h_{0}(t)=1, \quad 0 \leq t<1, \quad h_{1}(t)= \begin{cases}1, & 0 \leq t<0.5 \\ -1, & 0.5 \leq t<1\end{cases}
$$

An arbitrary signal $x(t) \in L^{2}[0,1)$ can be expanded by haar wavelet, i.e.,

$$
x(t)=\sum_{i=0}^{\infty} c_{i} h_{i}(t)
$$

where the Haar coefficients $c_{i}, i=0,1,2, \cdots$, are determined by $c_{i}=2^{j} \int_{0}^{1} x(t) h_{i}(t) d t$.

In practice, only the first $N$ terms of Eq. (8) are considered, where $N$ is a power of 2 . So we have

$$
x(t) \approx \sum_{i=0}^{N-1} c_{i} h_{i}(t)=C_{N}{ }^{T} H_{N}(t)=\hat{x}(t)
$$

then the N-square Haar matrix $\Psi_{N \times N}$ can be defined by

$$
\Psi_{N \times N} \triangleq\left[H_{N}\left(\frac{1}{2 N}\right) \quad H_{N}\left(\frac{3}{2 N}\right) \quad \ldots \quad H_{N}\left(\frac{2 N-1}{2 N}\right)\right] .
$$

$N$-term Block pulse functions can be written as following

$$
\Phi_{N}(t)=\left[\varphi_{1}, \varphi_{2}, \cdots, \varphi_{N}\right]^{T}
$$

According to Ref.[18,19], we can write the Block Pulse operational matrix of the fractional order integration $F^{\alpha}$ as following

$$
\left(I^{\alpha} \Phi_{N}\right)(t) \approx F^{\alpha} \Phi_{N}(t)
$$

where

$$
F^{\alpha}=\frac{1}{N^{\alpha}} \frac{1}{\Gamma(\alpha+2)}\left[\begin{array}{ccccc}
1 & \xi_{1} & \xi_{2} & \cdots & \xi_{N-1} \\
0 & 1 & \xi_{1} & \cdots & \xi_{N-2} \\
0 & 0 & 1 & \cdots & \xi_{N-3} \\
0 & 0 & 0 & \ddots & \vdots \\
0 & 0 & 0 & 0 & 1
\end{array}\right] .
$$

with $\xi_{k}=(k+1)^{\alpha+1}-2 k^{\alpha+1}+(k-1)^{\alpha+1}$.

Let $\left(I^{\alpha} H_{N}\right)(t) \approx P_{N \times N}^{\alpha} H_{N}(t)$, where the $N$-square matrix $P_{N \times N}^{\alpha}$ is called the Haar wavelet operational matrix of the fractional order integration. Then the Haar wavelet operational matrix of the fractional order integration $P_{N \times N}^{\alpha}$ is given by

$$
P_{N \times N}^{\alpha}=\Psi_{N \times N} F^{\alpha} \Psi_{N \times N}^{-1} .
$$

\section{IDENTIFICATION METHOD BASED ON HAAR WAVELET OPERATIONAL MATRIX}

Considering the fractional order nonlinear system described by (1), the goal of the system identification is to determine parameters $C, k, \lambda$ and the differential order $\alpha$ by the measured input and output data.

In order to obtain the output response of the identified model (1), the operational matrix method is used to solve the fractional order nonlinear differential equation.

Assuming the output response $y(t)$ and the excitation signal $f(t)$ are represented by Haar wavelets, i.e.:

$$
y(t) \approx Y^{T} H_{N}(t), f(t) \approx F^{T} H_{N}(t) .
$$

Applying the integral of order 2 to both sides of (1), one can obtain:

$$
y(t)+c I^{2-\alpha} y(t)+k I^{2} y(t)+\lambda I^{2} y^{3}(t)=I^{2} f(t) .
$$

Then substituting (15) into (16) gives:

$$
\begin{aligned}
& Y^{T} H_{N}(t)+c Y^{T} P_{N \times N}^{2-\alpha} H_{N}(t)+k Y^{T} P_{N \times N}^{2} H_{N}(t)+ \\
& \lambda I^{2}\left[Y^{T} H_{N}(t)\right]^{3}=F^{T} P_{N \times N}^{2} H_{N}(t) .
\end{aligned}
$$

In order to compute the integral of the nonlinear term $I^{2}\left[Y^{T} H_{N}(t)\right]^{3}$, Haar wavelets can be expanded in $H_{N}(t)=\Psi_{N \times N} \Phi_{N}(t)$. Then we have 


$$
\left[Y^{T} H_{N}(t)\right]^{2}=Y^{T} \Psi_{N \times N} \Phi_{N}(t)\left[\Phi_{N}(t)\right]^{T}\left[\Psi_{N \times N}\right]^{T} Y
$$

Setting $Y^{T} \Psi_{N \times N}=\left[\begin{array}{llll}w_{1} & w_{2} & \cdots & w_{N}\end{array}\right]$, from

$$
\Phi_{N}(t)\left[\Phi_{N}(t)\right]^{T}=\left[\begin{array}{llll}
\varphi_{1}(t) & & & 0 \\
& \varphi_{2}(t) & & \\
& & \ddots & \\
0 & & & \varphi_{N}(t)
\end{array}\right] .
$$

we have $\left[Y^{T} H_{N}(t)\right]^{2}=\left[\begin{array}{llll}w_{1}^{2} & w_{2}^{2} & \cdots & w_{N}^{2}\end{array}\right] \Phi_{N}(t)$.

Similarly, one obtains

$$
\left[Y^{T} H_{N}(t)\right]^{3}=\left[\begin{array}{llll}
w_{1}^{3} & w_{2}^{3} & \cdots & w_{N}^{3}
\end{array}\right] \Phi_{N}(t)
$$

namely $\left[Y^{T} H_{N}(t)\right]^{3}=\left[\begin{array}{llll}w_{1}^{3} & w_{2}^{3} & \cdots & w_{N}^{3}\end{array}\right]\left[\Psi_{N \times N}\right]^{-1} H_{N}(t)$

So, Eq. (17) can be rewritten as

$$
\begin{aligned}
& {\left[Y^{T}+c Y^{T} P_{N \times N}^{2-\alpha}\right]+} \\
& \left\{k Y^{T}+\lambda\left[\begin{array}{llll}
w_{1}^{3} & w_{2}^{3} & \cdots & w_{N}^{3}
\end{array}\right]\left[\Psi_{N \times N}\right]^{-1}-F^{T}\right\} P_{N \times N}^{2}=0 .
\end{aligned}
$$

Once $c, k, \lambda$, and the fractional differential order $\alpha$ are given, we can find the corresponding $Y$ by solving the nonlinear equation system. Here, "fsolve" is a standard function in the MATLAB used to solve this nonlinear equation system (21). Since $y(t) \approx Y^{T} H_{N}(t)$, we can obtain the output response $y(t)$. However, (21) is a nonlinear equation system, finding the output response $y(t)$ is needed much time. In fact, in system identification, our purpose is to obtain the estimation of parameters: $c, k, \lambda$ and $\alpha$ from the input $f(t)$ and output $y(t)$ of system. That's to say, we do not need to find $y(t)$ in the identification process. We can directly use the input and output signal of the system to identify parameters: $C, k, \lambda$ and $\alpha$. In order to avoid high time-consuming of the identification process, we use the least-squares method to find parameters $C, k$ and $\lambda$ by fixing the fractional derivative order $\alpha$. Then we vary the derivative order $\alpha$ in a interval to find the optimal parameters of $c, k$ and $\lambda$. For a fixed $\alpha$, the parameters $C, k$ and $\lambda$ can be determined by

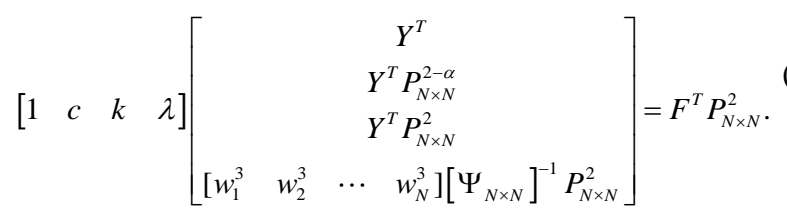

Then we use the following assessment criteria to the optimal fractional derivative order $\alpha$.

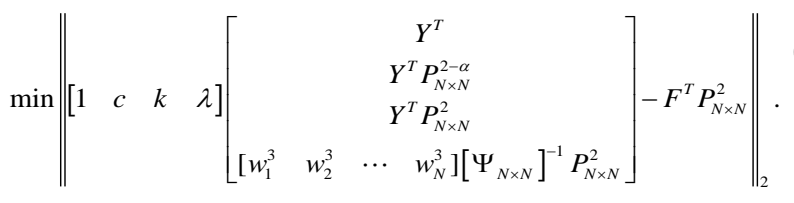

Thus, this nonlinear optimal problem is transformed into a linear optimal problem.

In additional, we can also take use of the multi-resolution property of the wavelet to fasten the identification process. Assuming the output response $y(t)$ and the excitation signal $f(t)$ are represented by $N=2^{J}$ terms Haar wavelets, their approximation can be represented by the first $N_{1}=2^{J-1}$ terms Haar wavelets and they also satisfy the (18). Thus, the dimension of (18) will reduce N/2 dimensions. As a result, we not only can fasten the identification process but also can reduce the noise affect. Thus, using the multi-resolution property of the wavelet, we can fasten the identification process.

\section{VerificAtion Of the Proposed Method}

To verify the effectiveness of the proposed method, two kinds of model are considered in this section. One is the Duffing oscillator and the other is the Riccati system.

\section{A. The First Model}

The Duffing oscillator is described by

$$
{ }_{0} D_{t}^{2} y(t)+c_{0} D_{t}^{\alpha} y(t)+k y(t)+\lambda y^{3}(t)=f(t), t>0
$$

with initial conditions: $y(0)=0, y^{\prime}(0)=0$. In (24), $c, k, \lambda$ and the differential order $\alpha$ are parameters which need to be identified. $f(t)$ is the excitation signal shown in Figure .1, and $y(t)$ is corresponding output response. The details of parameters are shown in Table 1 . Under no noise case, the output responses of the true and the identified system are given in Figure 2. In this case, $\mathrm{N}$ is 128.

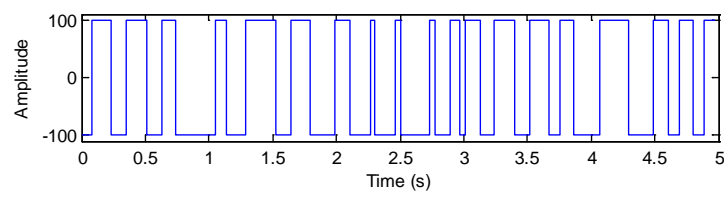

FIGURE I. EXCITATION SIGNAL

TABLE I. REAL PARAMETERS AND IDENTIFIED PARAMETERS OF THE DUFFING OSCILLATOR UNDER NO NOISE CASE

\begin{tabular}{ccccccccc}
\hline & \multicolumn{4}{c}{ Real Model } & \multicolumn{5}{c}{ Identified Model } \\
\cline { 2 - 9 } & $\alpha$ & $c$ & $k$ & $\lambda$ & $\tilde{\alpha}$ & $\tilde{c}$ & $\tilde{k}$ & $\tilde{\lambda}$ \\
\hline $\begin{array}{c}\text { Model } \\
1 \_1\end{array}$ & 1.2 & 0.3 & 800 & 0.7 & 1.2 & 0.30 & 800.00 & 0.70 \\
\hline $\begin{array}{c}\text { Model } \\
1 \_2\end{array}$ & 0.5 & 0.2 & 700 & 0.15 & 0.5 & 0.20 & 700.00 & 0.15 \\
\hline
\end{tabular}




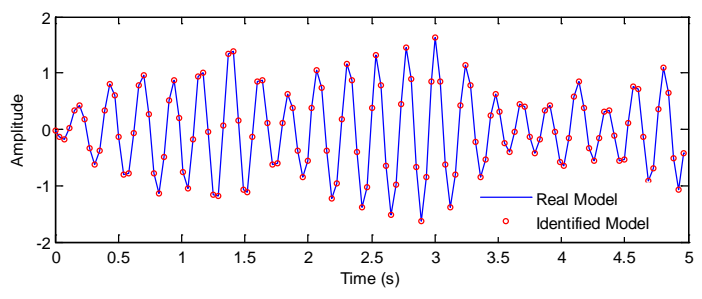

(a) Comparison the real model and the identified model for the model 1_1

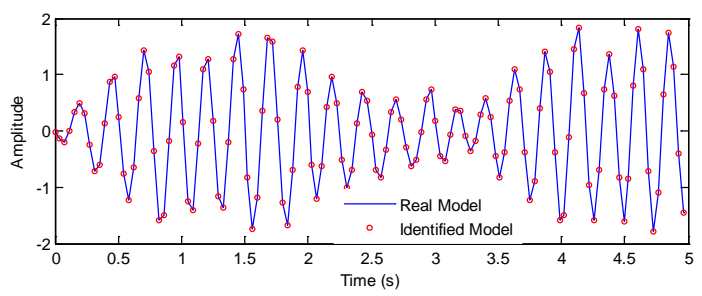

(b) Comparison the real model and the identified model for the model 1_2

FIGURE II. OUTPUT RESPONSES OF THE REAL MODEL AND THE IDENTIFIED MODEL FOR THE DUFFING OSCILLATOR

B. The Second Model

Riccati system [9]:

$$
{ }_{0} D_{t}^{\alpha} y(t)-k y(t)+\lambda y^{3}(t)=f(t), \quad 0<\alpha \leq 2
$$

with initial condition $y^{(n)}(0)=0, n=0,1, \cdots,\lfloor\alpha\rfloor$.

where $k, \lambda$ and the differential order $\alpha$ are parameters needed to be identified. $f(t)$ is the excitation signal, and $y(t)$ is the corresponding output response. Under no noise case, the identification results are presented in Table 2.

TABLE II. IDENTIFICATION RESULTS WITHOUT NOISE FOR RICCATI SYSTEM

\begin{tabular}{ccccccc}
\hline & \multicolumn{3}{c}{ Real model } & \multicolumn{3}{c}{ Identified model } \\
\cline { 2 - 7 } & $\alpha$ & $k$ & $\lambda$ & $\tilde{\alpha}$ & $\tilde{k}$ & $\tilde{\lambda}$ \\
\hline Model 2_1 & 1 & 2.0 & 1.0 & 1.0 & 2.0000 & 1.0000 \\
Model 2_2 & 0.9 & 2.0 & 1.0 & 1.0 & 2.0000 & 1.0000 \\
Model 2_3 & 1.5 & 2.0 & 1.0 & 1.5 & 2.0000 & 1.0000 \\
\hline
\end{tabular}

In order to verify the proposed method is robust to noise, the riccati systems are identified again under noise case. At first, we generate the input signal $f(t)$ and output signal $y(t)$, the length of signal is $\mathrm{N}=1024$. Then we resample the input and output signal and use them to identify the riccati systems, the length of signal is $\mathrm{N}=512, \mathrm{~N}=256$ and $\mathrm{N}=128$, respectively. The identified results of model 2_3 are shown in Table 3. One can see that the proposed method can obtain the desired results using the signal of the resampling input and output. The optimal fractional order is determined by the assessment criteria Eq. (21).

TABLE III. IDENTIFIED RESULTS BY RESAMPLING THE INPUT AND OUTPUT OF RICCATI SYSTEM (MODEL 2_3)

\begin{tabular}{ccccc}
\hline \multirow{2}{*}{ Real Model } & \multicolumn{4}{c}{ Identified Model } \\
\cline { 2 - 5 } & $\mathrm{N}=1024$ & $\mathrm{~N}=512$ & $\mathrm{~N}=256$ & $\mathrm{~N}=128$ \\
\hline 1.5 & 1.5 & 1.5 & 1.5 & 1.5 \\
2.0 & 2.0074 & 1.9946 & 2.0125 & 2.0624 \\
1.0 & 1.0022 & 0.9985 & 1.0022 & 1.0077 \\
\hline
\end{tabular}

In additional, we also consider the identification of the riccati system under noise case. The excitation signal shown in Figure $.3(\mathrm{a})$, the signal length is $\mathrm{N}=1024$. The response signal is added white noise and the signal to noise ratio (SNR) is $20 \mathrm{~dB}$. The signal and the identification result are shown in Figure 3 (b).

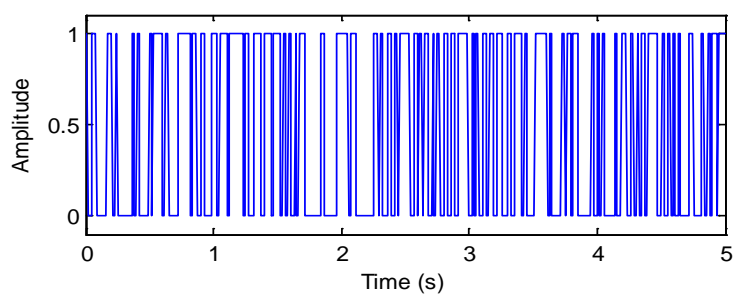

(a) Excitation signal

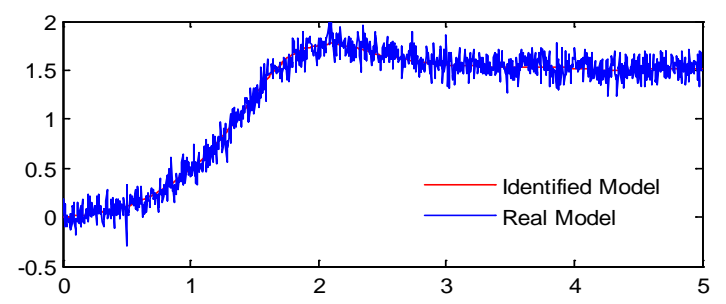

(b) Response of the real model and the identified model for the Riccati system (model 2_3)

FIGURE III. IN THE CASE OF 20DB, IDENTIFIED RESULT BY USING
WAVELET METHOD

\section{CONCLUSION}

Through the application of the Haar wavelet operational matrix of the fractional order integration, a system was proposed. Numerical simulations, involving both integral and fractional and order systems, confirm the efficiency of the above methodology. An advantage of the proposed method lies in fastening the identification process by using the multiresolution nature of the wavelet.

\section{ACKNOWLEDGMENT}

The work was partly supported by outstanding innovation team project of universities in Jiangsu province, National Natural Science Foundation of China (Grant: 61671010, 61271395), and the Natural Science Foundation of Jiangsu Province of China (Grant: BK20161513). 


\section{REFERENCES}

[1] Machado JT. Optimal tuning of fractional controllers using genetic algorithms. Nonlinear Dynamics. 2010;62:447-52.

[2] Calderón AJ, Vinagre BM, Feliu V. Fractional order control strategies for power electronic buck converters. Signal Processing. 2006;86:28032819.

[3] Dorcak L. Numerical models for the simulation of the fractional-order control systems. arXiv preprint math/0204108. 2002.

[4] Shen Y, Yang S, Xing H, Gao G. Primary resonance of Duffing oscillator with fractional-order derivative. Communications in Nonlinear Science and Numerical Simulation. 2012;17:3092-100.

[5] Raynaud H-F, Zergainoh A. State-space representation for fractional order controllers. Automatica. 2000;36:1017-21.

[6] Tang Y, Liu H, Wang W, Lian Q, Guan X. Parameter identification of fractional order systems using block pulse functions. Signal Processing. 2014.

[7] Li Y. Solving a nonlinear fractional differential equation using Chebyshev wavelets. Communications in Nonlinear Science and Numerical Simulation. 2010;15:2284-92

[8] Saha Ray S. On Haar wavelet operational matrix of general order and its application for the numerical solution of fractional Bagley Torvik equation. Applied Mathematics and Computation. 2012;218:5239-48

[9] Momani S, Shawagfeh N. Decomposition method for solving fractional Riccati differential equations. Applied Mathematics and Computation. 2006;182:1083-93 